University of Warwick institutional repository: http://go.warwick.ac.uk/wrap This paper is made available online in accordance with publisher policies. Please scroll down to view the document itself. Please refer to the repository record for this item and our policy information available from the repository home page for further information.

To see the final version of this paper please visit the publisher's website. Access to the published version may require a subscription.

Author(s): Maughan, Barbara and Carroll, Julia

Article Title: Literacy and mental disorders

Year of publication: 2006

Link to published version:

http://dx.doi.org/10.1097/01.yco.0000228752.79990.41

Publisher statement: None 


\section{Literacy and mental disorders}

Barbara Maughan $^{1}$ and Julia Carroll ${ }^{2}$

\footnotetext{
${ }^{1}$ MRC Social, Genetic and Developmental Psychiatry Centre
} King's College London Institute of Psychiatry

\footnotetext{
${ }^{2}$ Department of Psychology, University of Warwick
} 
Word count: 2515

Correspondence to Barbara Maughan, MRC Social, Genetic and Developmental Psychiatry Centre, Box PO46, King’s College London Institute of Psychiatry, De Crespigny Park, London SE5 8AF. Tel: +44 (0) 20-7848-0470; e-mail b.maughan@iop.kcl.ac.uk 


\section{Structured abstract}

\section{Purpose of review}

This review examines recent evidence on the comorbidity between literacy problems and psychiatric disorder in childhood, and discusses possible contributory factors.

\section{Recent findings}

Recent studies confirm the substantial overlap of literacy problems with a range of emotional/behavioural difficulties in childhood. Literacy problems and inattention may have a shared genetic infuence, thus creating the link with attention-deficit hyperactivity disorder. To an extent, links between literacy problems and conduct problems may also be mediated by attentional difficulties. In addition, findings suggest bidirectional influences whereby disruptive behaviours impede reading progress and reading failure exacerbates risk for behaviour problems. The association between literacy problems and anxiety disorders is not mediated by inattentiveness, rather, anxiety arises from the stressors associated with reading failure. Findings in relation to depression are less consistent, but suggest that poor readers may be vulnerable to low mood. Children with autism seem more likely to face problems in reading comprehension rather than the decoding difficulties that are more prominent in other disorders.

\section{Summary}

Literacy problems are associated with externalizing and internalizing disorders in childhood, with different mechanisms likely to be implicated in each case. When comorbid problems occur, each is likely to require separate treatment. 
Keywords

dyslexia, mental disorders, comorbidity 


\section{Introduction}

Literacy problems are common in childhood, and show strong persistence throughout development. Prevalence estimates vary according to the definition used, but studies suggest that between 5\% and 10\% of children in English-speaking countries face severe and specific difficulties in learning to read. Almost inevitably, such problems impact on children's school experiences, restrict educational attainments and affect later life chances. In addition, it has long been clear that children with literacy problems are at increased risk of psychiatric disorder. Comorbidity with disruptive disorders has been most extensively reported [1]; though less frequently studied, past evidence also suggests that poor readers show higher than expected rates of emotional difficulties [2].

These findings raise key questions about the reasons for the overlap of reading and psychiatric disorders; about the impact of such overlap on the course of both literacy problems and emotional/behavioural difficulties; and about implications for treatment. Each of these topics has received some attention in recent research, though progress in some arenas - most notably in relation to associations with attention deficit hyperactivity disorder (ADHD) - has been more extensive than developments in others. We report here on findings published since 2004. Our review falls into three parts. First, as a background, we provide a brief overview of current findings on reading disabilities and the cognitive, genetic and neurobiological factors thought to underlie them. Second, we report recent evidence on general patterns of psychiatric comorbidity in children and adolescents with reading difficulties, and possible bases for these increased risks. Third, we focus on comorbidity with specific disorders, and outline progress in understanding causes of these overlaps. 


\section{Developmental reading problems}

Successful reading requires both accurate word decoding and comprehension; reading difficulties can arise from problems in either or both of these domains. Unexpected decoding problems among children with adequate intellectual skills and opportunities to learn (usually referred to as dyslexia, or specific reading difficulties) are the most well documented basis for developmental reading problems [3*]. More recently, comprehension problems in children with good decoding skills have also been investigated [4*]. Extensive cognitive research has confirmed that phonological difficulties (problems in understanding the sound structure of speech), are the major proximal cause of dyslexia [5]. The most consistent finding from neuroimaging studies of individuals with reading problems is reduced volume in specific focal brain regions mediating speech and learning, and abnormal activation patterns in response to linguistic stimuli [3*]. Although reading difficulties per se only become apparent when children are exposed to reading instruction, pre-schoolers who go on to develop dyslexia may be slow to acquire oral language skills. Evidence is emerging that brain responses to speech sounds can distinguish at risk groups at young ages [6]. Family and twin studies show that dyslexia is both familial and heritable. Genetically informative designs are now being used to delineate genetic and environmental influences on constituent skills in preschool and early school age children $[7,8]$. Replicated linkage studies implicate loci on chromosomes 2, 3, 6, 15 and 18, and progress is being made in identifying more specific genetic variations that may affect early brain development [9]. Rates of developmental reading problems are also associated with indicators of family and social disadvantage, underscoring the role of environmental risks. 


\section{Comorbidity between literacy difficulties and psychiatric disorders: overview}

Findings from the 1999 ONS British Child Mental Health Survey provide the most comprehensive evidence to date on the overall patterns of comorbidity between specific literacy difficulties and emotional/behavioural problems in childhood and adolescence. In a large, nationally representative sample of 9-15 year-olds, Carroll et al. [10**] found that literacy problems showed significant associations with each of the major disorder categories except depression, with odds ratios ranging from 2.7 (95\% confidence interval [CI] 1.8-4.3) for anxiety disorders to 3.8 (95\% CI 2.4-6.1) for ADHD. Dimensional analyses showed that these associations were not solely confined to the small proportions of children who meet formal criteria for psychiatric disorder. Literacy problems were associated with higher rates of parent-rated emotional/behavioural difficulties, and with adolescents' own reports of low mood, even among children who did not show clinically significant difficulties. Though girls were less likely to show literacy problems than boys, relative risks for psychiatric disorders and more broad range emotional/behavioural difficulties did not vary between the sexes.

Conceptual discussions have now set out a range of factors, both artefactual and substantive, that might contribute observed comorbidity between disorders [11]. Methodological artefacts can generally be ruled out in studies of reading and behaviour problems, as each difficulty is assessed quite independently. Sampling issues may be more problematic. Although patterns of comorbidity have been well described in community samples, hypothesized contributory deficits are often initially examined in clinic populations, which are vulnerable to referral bias. In addition, samples are sometimes too small to permit adequate examination of the potential causes of comorbidity [12]. 
Among substantive reasons for comorbidity, three possibilities have attracted most attention: (i) that the two disorders share common risk factors, whether neurobiological, psychological, genetic or social; (ii) that one disorder increases risk for the other (so that, for example, behaviour problems may impede reading acquisition, or frustrations arising from learning difficulties may exacerbate risk for behaviour problems); or (iii) that the comorbid condition is a separate entity, distinct from either 'pure' disorder. Because of the broad spectrum of emotional/behavioural problems identified among poor readers, investigators have also considered a fourth possibility: that some of these bivariate links may be epiphenomenal, reflecting comorbidities among other disorders [13]. ADHD and its sub-types have received particular attention as possible 'core' comorbid conditions in this context.For this reason we begin our review of recent findings on associations with individual disorders by considering evidence in relation to ADHD.

\section{ADHD}

ADHD is the most frequent psychiatric concomitant of reading disabilities in childhood. Past studies suggest that between $25 \%$ and $40 \%$ of children with ADHD meet criteria for reading disorders, and that $15-40 \%$ of those with reading difficulties meet criteria for ADHD. Consistent with prior studies, Carroll et al. [10**] found that these associations primarily reflected elevated levels of inattentive symptoms among children with reading problems. Poor readers showed no excess of overactivity. A proposal that inattentiveness among poor readers might simply be a phenocopy of ADHD has been rejected, Willcutt et al. [14*], examined neuropsychological deficits in the two disorders and found that although problems in phonological processing were specific to poor readers, no deficits were uniquely associated with ADHD. Comorbid cases showed an additive combination of the weaknesses 
found in each 'pure' group. Banaschewski et al. [15*] reviewed evidence on the neuropsychological, neurobiological and genetic correlates of ADHD and a range of comorbid disorders. They concluded that many components of the aetiological pathways to ADHD may be shared with other conditions. Spira and Fischel [16*] reviewed evidence for four possible models of the association between pre-school inattention and literacy development, including shared risks, unidirectional causation, and bi-directional links. Evidence for exclusively one-way causal associations seemed minimal. Instead, these authors argued that current findings provide strong intuitive support for complex multidirectional linkages across development. Treatment studies suggest that inattention compromises poor readers' responses to reading instruction [17], and that multimodal treatment for ADHD, though reducing risks of other comorbid conditions, does not markedly affect rates of reading disability [18]. As a result, each condition appears to require specific interventions.

All recent reviews have also highlighted growing evidence for a common genetic basis for associations between ADHD and reading difficulties. Both disorders are heritable, and bivariate twin analyses suggest that comorbidity between the two conditions is primarily due to common genetic influences. Bivariate linkage studies have now begun to search for chromosomal regions containing genes that increase risk for both disorders. The first genome-wide linkage analysis of measures of reading ability in children with ADHD [19] supported the existence of genetic factors that have pleiotropic effects on ADHD and reading ability, along with others unique to reading. Gayan et al. [20**] carried out the first bivariate linkage analysis of ADHD and reading disability, and identified several gene areas that seemed to have multiple effects, most notably on chromosomes 14 and 16 . Interestingly, several loci previously highlighted in univariate analyses of ADHD or reading disabilities did 
not show significant bivariate effects, suggesting that some genes are specific to particular disorders. Following neurobiological evidence implicating noradrenergic function in the aetiology of ADHD comorbid with reading difficulties, Stevenson et al. [21**] provided preliminary evidence, in an ADHD sample, of associations of reading problems with the alpha 2A adrenergic receptor (ADRA2A) polymorphism. Findings of this kind represent the first steps on a path that should in time contribute to improvements in the early identification of at-risk children, and may even provide the basis for more individually tailored approaches to intervention.

\section{Conduct problems}

In addition to ADHD, poor readers are also at increased risk of other disruptive disorders. The Isle of Wight epidemiological studies of the 1960s [22] were among the first to note these links: in 9/10 year-olds, a third of children with specific reading difficulties met criteria for conduct disorder, and a third of those with conduct disorder had specific reading problems. Thirty years on, Carroll et al. [10**] documented a three-fold increase in risk for Conduct/Oppositional Defiant Disorders among 9-15 year olds with literacy problems in the UK national sample, and Arnold et al. [23*] confirmed numerous past reports of elevated levels of more broadly defined measures of conduct problem in the mid-late teens.

Because conduct problems often co-occur with ADHD, some of these links may be epiphenominal, attributable to the common feature of inattentiveness. Empirical evidence here is varied. Not all studies find that inattentiveness mediates the association between reading difficulties and disruptive behaviours. In terms of shared environmental risks, Carroll et al. [10**] concluded that family stressors were unlikely to be key contributors.: 
Although literacy problems were associated with family class, they showed no links with markers of parental psychopathology or family dysfunction frequently implicated in risk for behaviour problems. Trzesniewski et al. [24**] provide the first genetically informed analyses of reading and antisocial behaviour, based on a longitudinal study of twins assessed at ages 5 and 7 years. Where reading-ADHD associations were best explained by common genetic influences, twin analyses of reading-conduct problem links in boys highlighted a main role for environmental factors common to both. Leading candidate environmental risks only weakly mediated these associations, while a reciprocal causation model (poor reading achievement increasing risks of antisocial behaviour, and conduct problems impeding reading progress) offered the best account of the findings. Previous studies have suggested that reciprocal processes of this kind can occur throughout schooling, contributing in the later years to increased risks of both truancy and delinquency. Pre-school programmes [25] and school-based mastery learning interventions are associated with reduced risks of later aggression and delinquency; effective interventions for behaviour problems have not as yet been found to affect reading achievement.

\section{Anxiety and depression}

Although disruptive disorders have formed the prime focus of research on comorbidity with reading difficulties, findings have also identified elevated risks of emotional problems.

Carroll et al. [10**] found increased rates of both generalized anxiety and separation anxiety disorder among 9-15 year-olds with reading difficulties, but no excess of specific phobias. Importantly, these risks were not attributable to inattentiveness: associations between reading problems and anxiety disorders remain significant with inattention scores controlled. Arnold et al. [23**] reported a similar pattern in a 3-year longitudinal study in the teens. Carroll and 
Iles [26] found elevated levels of both state and trait anxiety among dyslexic students in higher education.; Importantly, these difficulties were not limited to concerns about academic situations, but were also reflected in measures of social anxiety. As yet, very little is known about the processes underlying these links. Prior studies [4*] suggest that associations between reading difficulties and anxiety are not attributable to shared family risks. Instead, commentators have speculated that education-related stressors may play a key role. Because parents may not be aware of internalizing problems [23*], teachers and other education professionals may be in an especially important position to identify such difficulties and ensure appropriate management.

Findings in relation to depression are more varied. Carroll et al. [10**] found no excess of depressive disorders among 9-15 year-old poor readers, and that elevations in adolescents' own reports of depressed mood (primarily evident among younger teenage boys) were attributable to associated inattentiveness. Among older teenagers, Arnold et al. [23**] also found that self (but not parent) reports of depressed mood were elevated among poor readers. Comorbid ADHD did not appear to account for the links. A meta-analysis [27] concluded that rates of low mood, but not of clinically significant depressive disorders, may be elevated among more broadly defined learning disabled samples, possibly associated with low selfesteem and exposure to teasing and bullying by peers [28]. All recent commentators have noted the need for further research into associations between reading and depression to clarify inconsistencies in current findings.

\section{Autism and other developmental disorders}


previous descriptive research suggested that children with autism may have word reading skills in advance of their reading comprehension (hyperlexia).[29]. Findings from recent systematic research are contradictory. Nation et al. [30*] examined the reading of single words, nonsense words and connected text in a relatively large sample of children with an autism spectrum disorder. While a subset had no measurable reading ability, the remainder showed generally average levels of reading accuracy for their age, but poor reading comprehension.

Nation and Norbury $\left[4^{*}\right]$ examined skills in three groups of children thought to have poor reading comprehension - specific poor comprehenders, children with autism and children with specific language impairment. All three groups show reading comprehension close to their level of language skills, suggesting that language development is the main determinant of reading comprehension.

Reading skills are in large part dependent upon oral language skills, with word reading largely dependent on a child's phonological skills and reading comprehension dependent upon language comprehension. Intervention studies do suggest, however, that for some autistic children for whom word reading is a relative strength, this skill can be used to improve language development [31].

\section{Conclusions}

Recent studies have extended our understanding of both the extent and some of the likely causes of poor readers' increased vulnerability to emotional and behavioural problems. In some areas most notably in relation to comorbidity with ADHD - detailed behavioural, neuropsychological 
and genetic research in each constituent disorder has provided a strong basis for identifying contributors to the overlaps. In others - most notably in relation to emotional difficulties and to autism - evidence is still limited, and models of underlying processes largely speculative. Current findings suggest, however, that somewhat different mechanisms are likely to be implicated in poor readers' increased vulnerability to ADHD, to conduct problems and to emotional disorder and distress. Data from intervention studies remain sparse. Recent reports appear to confirm, however, that where comorbid literacy and emotional/behavioural problems are detected, effective treatments in one domain will not necessarily result in improvements in the other; as a result, each disorder needs treating separately.

\section{References}

1. Hinshaw SP. Externalizing behavior problems and academic underachievement in childhood and adolescence: causal relationships and underlying mechanisms. Psychol Bull 1992; 111:127-155.

2. Willcutt EG, Pennington BF. Psychiatric comorbidity in children and adolescents with reading disability. J Child Psychol Psychiatry 2000; 41:1039-1048.

*3. Shaywitz SE, Shaywitz BA. Dyslexia (Specific Reading Disability). Biol Psychiatry 2005; 57:1301-1309. A review of research into the brain structures and processes associated with dyslexia.

*4. Nation K, Norbury CF. Why reading comprehension fails. Topics in Language Disorders 2005; 25:21-32. A comparison of children with language impairment, autism and specific comprehension difficulties, suggesting that language is the major mediating factor in reading comprehension.

5. Vellutino FR, Fletcher JM, Snowling MJ, et al. Specific reading disability (dyslexia): what have we learned in the past four decades? J Child Psychol Psychiatry 2004; 45:2-40. 
6. Lyytinen H, Guttorm TK, Huttunen T, et al. Psychophysiology of developmental dyslexia: a review of findings including studies of children at risk for dyslexia. Journal of Neurolinguistics 2005; 18:167-195.

7. Byrne B, Wadsworth S, Corley R, et al. Longitudinal twin study of early literacy development: Preschool and kindergarten phases. Scientific Studies of Reading 2005; 9:219-235.

8. Oliver BR, Dale PS, Plomin R. Predicting literacy at age 7 from preliteracy at age 4 - A longitudinal genetic analysis. Psychol Sci 2005; 16:861-865.

9. Miller G. Genes that guide brain development linked to dyslexia.. Science 2005; 310:759.

**10. Carroll J, Maughan B, Goodman R, Meltzer H. Literacy difficulties and psychiatric disorders: evidence for comorbidity. J Child Psychol Psychiatry 2005; 46: 524-532.

A large-scale, epidemiological study based on a nationally representative sample documenting the extent of comorbidity between dyslexia and a range of psychiatric disorders, and exploring likely mediating mechanisms.

11. Neale M.C, Kendler, KS. Models of comorbidity for multifactorial disorders. Am J Hum Genet 1995; 54:935945.

12. Rhee SH, Hewitt JK, Corley RP, et al. Testing hypotheses regarding the causes of comorbidity: Examining the underlying deficits of comorbid disorders. J Abnorm Psychol 2005; 114:346-362.

13. Angold A, Costello EJ, Erkanli A. Comorbidity. J Child Psychol Psychiatry 1999; 40:57-87.

*14. Willcutt EG, Pennington BF, Olson RK, et al. Neuropsychological analyses of comorbidity between reading disability and attention deficit hyperactivity disorder: In search of the common deficit. Dev Neuropsychol 2005; 27:35-78.

Neuropsychological profiles of children with comorbid ADHD and reading disability reflect an additive combination of the deficits shown by each group. 
*15. Banaschewski T, Hollis C, Oosterlaan J, et al. Towards an understanding of unique and shared pathways in the psychopathophysiology of ADHD. Dev Sci 2005; 8:132-140.

Review of neuropsychological, neurobiological and genetic correlates of ADHD and comorbid disorders.

*16. Spira EG, Fischel JE. The impact of preschool inattention, hyperactivity, and impulsivity on social and academic development: a review. J Child Psychol Psychiatry 2005; 46:755-773.

Review examining progression of core symptoms of ADHD through the preschool years. Though symptoms of overactivity are recognisable earlier in development than inattention, the latter is more closely related to academic achievement.

17. Rabiner DL, Malone PS and the Conduct Problems Prevention Res Group. The impact of tutoring on early reading achievement for children with and without attention problems. J Abnorm Child Psychol 2004; 32:273-284.

18. Hechtman L, Etcovitch J, Platt R, et al. Does multimodal treatment of ADHD decrease other diagnoses? Clin Neurosci Res 2005; 5:273-282.

19. Loo SK, Fisher SE, Francks C, et al. Genome-wide scan of reading ability in affected sibling pairs with attentiondeficit/hyperactivity disorder: unique and shared genetic effects. Mol Psychiatry 2004; 9:485-493.

**20. Gayan J, Willcutt EG, Fisher SE, et al. Bivariate linkage scan for reading disability and attentiondeficit/hyperactivity disorder localizes pleiotropic loci. J Child Psychol Psychiatry 2005; 46:1045-1056.

The first genome-wide bivariate linkage analysis of reading disability and ADHD in sibling pairs in which at least one has a reading difficulty, showing evidence for bivariate linkage on chromosomes 14 and 13.

**21. Stevenson J, Langley K, Pay H, et al. Attention deficit hyperactivity disorder with reading disabilities: preliminary genetic findings on the involvement of the ADRA2A gene. J Child Psychol Psychiatry 2005; 46:10811088.

Preliminary evidence, in an ADHD sample, that the ADRA2A gene may contribute to comorbidity with reading difficulties. 
22. Rutter M, Tizard J, Whitmore K. Education, Health and Behaviour. London: Longmans 1970. (reprinted 1981, Melbourne, FA: Krieger).

**23. Arnold EM, Goldston DB, Walsh AK, et al. Severity of emotional and behavioral problems among poor and typical readers. J Abnorm Child Psychol 2005; 33:205-217.

Longitudinal study of emotional and behavioural problems in poor and typically developing readers in adolescence. Both adolescents and parents described elevated levels of inattention and delinquent behaviours; adolescents, but not parents, reported increased levels of anxiety and depression.

**24. Trzesniewski K, Moffitt TE, Caspi A, et al. Revisiting the association between reading achievement and antisocial behavior: new evidence of an environmental explanation from a twin study. Child Dev (in press.) The first twin study of reading-antisocial behaviour comorbidity, focusing on 5-7 year-olds. In boys, associations were best accounted for by reciprocal associations.

25. Reynolds AJ, On SR, Topitzes JW. Paths of effects of early childhood intervention on educational attainment and delinquency: A confirmatory analysis of the Chicago Child-Parent Centers. Child Dev 2004; 75:1299-1328.

26. Carroll JM, Iles, JE. An assessment of anxiety levels in dyslexic students in higher education. British Journal of Educational Psychology, in press.

27. Maag JW, Reid R. Depression among students with learning disabilities: Assessing the risk. J Learn Disabil 2006; 39:3-10.

28. Singer E. The strategies adopted by Dutch children with dyslexia to maintain their self-esteem when teased at school J Learn Disabil 2005; 38:411-423.

29. Grigorenko EL, Klin A, Pauls DL, et al. A descriptive study of hyperlexia in a clinically referred sample of children with developmental delays. J Autism Dev Disord 2002; 32:3-12.

*30. Nation K, Clarke P, Wright B, Williams, C. Patterns of reading ability in children with autistic spectrum disorders. J Autism Dev Disord in press. 
Children with autism and measurable reading abilities showed generally average levels of reading accuracy, but poor reading comprehension. Reliance on tests of word reading may overestimate children's reading competence in other areas.

31. Craig HK, Telfer AS. (2005) Hyperlexia and autism spectrum disorder: A case study of scaffolding language growth over time. Topics in Language Disorders 2005; 25:364-374. 